\title{
Greenness, Plant Height and Yellow Berry of the Wheat Grain in Response to Paclobutrazol
}

\author{
Agustín Zárate Márquez¹, Juan Francisco Ponce Medina1, Leopoldo Partida Ruvalcaba2*, \\ Felipe Ayala Tafoya ${ }^{3}$, Moisés Gilberto Yáñez Juárez³, Teresa de Jesús Velázquez Alcaraz ${ }^{3}$, \\ Raymundo Medina López ${ }^{3}$ \\ ${ }^{1}$ Instituto de Ciencias Agrícolas de la Universidad Autónoma de Baja California, \\ Mexicali, México \\ ${ }^{2}$ Universidad Tecnológica de Culiacán, Culiacán de Rosales, México \\ ${ }^{3}$ Facultad de Agronomía de la Universidad Autónoma de Sinaloa, Culiacán, México \\ Email: [eopoldo partida@utculiacan.edu.mx
}

Received 24 June 2015; accepted 27 June 2015; published 30 June 2015

Copyright (C) 2015 by authors and OALib.

This work is licensed under the Creative Commons Attribution International License (CC BY).

http://creativecommons.org/licenses/by/4.0/

(c) (i) Open Access

\section{Abstract}

To know the response of the wheat in the greenness, plant height and grain with yellow berry, paclobutrazol (PBZ) was applied to the foliage of the cultivars "Cachanilla F-2000", "Yécora F-70" and

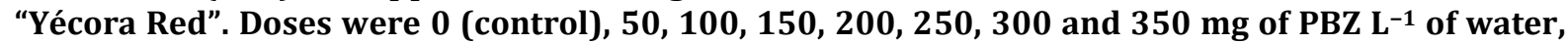
which were applied only once with a hand pump. The experimental design was a randomized complete block with four replications; experimental units of four rows of $20 \mathrm{~m}$ long, which were fertilized with $364 \mathrm{~kg} \cdot \mathrm{N} \cdot \mathrm{ha}^{-1}$ more $56 \mathrm{~kg}$ of $\mathrm{P}$, were applied in pre-seeding. In "Cachanilla F-2000" greenness, the maximum increase (7.2\%) was achieved with $100 \mathrm{mg}$ of $\mathrm{PBZ} \mathrm{L}^{-1}$ of water. In "Yécora F-70" was $12.2 \%$ with $300 \mathrm{mg}$, and in "Yécora Red" was $19.1 \%$ with $50 \mathrm{mg}$ of PBZ. In "Cachanilla F-2000" height decreased $6.0 \%, 6.3 \%, 6.3 \%, 9.4 \%, 10.7 \%, 10.9 \%$ and $13.0 \%$ with 50,100 , 150, 200, 250, 300 or $350 \mathrm{mg}$ of PBZ, compared with the control. In "Yécora F-70" the largest decrease $(10 \%)$ occurred with $150 \mathrm{mg}$ of PBZ, while in "Yécora Red" height decreases ranged from $7.9 \%-17.0 \%$ with doses of $50-350 \mathrm{mg}$. The yellow berry decreased from $48.5 \%-74.4 \%$ in "Cachanilla F-2000", and to zero in "Yécora F-70" and "Yécora Red". These results indicate that PBZ can be used to increase greenness and photosynthesis to improve the quality of the grain of flour and durum wheat.

\section{Keywords}

Growth Retardant, High, “Cachanilla F-2000”, "Yécora F-70”, “Yécora Rojo”

${ }^{*}$ Corresponding author. 


\section{Introduction}

El trigo (Triticum aestivum L.) es el cereal que actualmente ocupa el segundo lugar en importancia de volúmenes de producción mundial, con una tendencia a incrementarse desde el año 2004 con producciones por arriba de 588.6 millones de toneladas. En el período 1998-2008, la producción de trigo fue superior al consumo mundial; sin embargo, a partir del ciclo 2008-2009 la producción de trigo fue menor a lo que la población mundial necesitó para su consumo, como lo indican Ochoa \& Ortega [1].

En la última década, la demanda mundial de trigo se incrementó de manera considerable, en tanto que la producción no aumentó en la misma proporción, ya sea por los bajos niveles de productividad en algunas regiones o bien por problemas climáticos, lo que dio como resultado que las reservas mundiales del grano se vieran mermadas de manera importante, como lo informan Ochoa \& Ortega [1].

La estructura del paclobutrazol (PBZ) [(2RS, 3RS)-1-(4-clorofenil)-4,4-dimetil-2-(1h-1,2,4-triazol-1-il) pentan-3-ol] es similar a la del brasinazole; sin embargo, éste último es un potente inhibidor de la biosíntesis del brasinosteroide, clasificado como una nueva clase de fitohormona, según Clouse \& Sasse [2].

Buscando alternativas para mejorar calidad, crecimiento y desarrollo de plantas, en las dos décadas pasadas se descubrió que el PBZ es un regulador de crecimiento que incrementa el crecimiento de raíces, de acuerdo con Watson [3] o aumenta la relación de raíces por brotes en plantas de Prunus persica L. o durazno, según Litembani \& Taylor [4], así como en Pyracantha y Juniperus, informado por Ruter [5]. Sin embargo, esta sustancia tiene alta residualidad en el suelo, por lo que puede provocar contaminación de mantos freáticos y riesgo potencial de translocación en los frutos, no obstante, dicha residualidad depende de que se hagan aplicaciones consecutivas a través del tiempo, como lo mencionan Osuna et al. [6].

El PBZ es una sustancia que ocasiona efectos en el crecimiento de raíces y parte aérea de plántulas de pimiento morrón (Capsicum annuum) variedad “California Wonder” y de una población segregante de berenjena (Solanum melongena) varidad "Dalia”. El PBZ incrementa la biomasa de raíz y de la parte aérea de ambas especies; en la primera, la dosis de $150 \mathrm{mg}_{\text {de }} \mathrm{PBZ} \mathrm{L}^{-1}$ de agua puede incrementar hasta 1.1 veces la longitud de la raíces, 3.7 veces la materia fresca y 13 veces la materia seca de las mismas; pero también puede incrementar en 1.5 y 6.7 veces la materia fresca y seca de la parte aérea de la misma especie. No obstante, en berenjena puede incrementar en 1.3 veces la materia fresca y en $71 \%$ la materia seca de la raíz; asimismo, $81 \%$ la materia fresca y 89\% la materia seca de la parte aérea, como lo discuten Partida et al. [7].

Según Velázquez et al. [8], el PBZ también ocasiona efectos en la parte aérea de plántulas de tomate y chile, de tal forma que cuando se aplica sobre plántulas de tomate con dos o cuatro hojas verdaderas en dosis de 100 , 150 ó 200 mg de PBZ L ${ }^{-1}$ de agua, la altura de plantas se retarda; mientras que cuando se aplica en dosis de 250, 300 ó 350 mg de PBZ L ${ }^{-1}$ de agua, la altura se incrementa. Asimismo, en chile el PBZ retarda el crecimiento de los tipos chile bell y anaheim con $200 \mathrm{mg} \cdot \mathrm{L}^{-1}$, en jalapeño con 100, en serrano con 100 ó 200, y en caribe con 200 ó 250.

En pepino, el paclobutrazol ocasiona aumento en el número de raíces, longitud y diámetro de las mismas, cuando las semillas son remojadas en solución con $40 \mathrm{mg}$ de $\mathrm{PBZ} \mathrm{L}^{-1}$ de agua, pero la longitud del hipocótilo se reduce. Además, las plantas que resultan de semillas tratadas con paclobutrazol y refrigeración durante 4 días a $5^{\circ} \mathrm{C}$ tienen mayor concentración y fluorescencia de clorofila, por lo que son más eficientes fotosintéticamente en relación con las plantas testigo, como lo indica Ali [9].

En la región de Mexicali, Baja California, México, el cultivo de trigo ocupa el primer en superficie sembrada; sin embargo, algunas veces el grano de trigo no tiene la calidad requerida por la industria, debido a la presencia de alto porcentaje de granos con panza blanca, lo cual no afecta el rendimiento, así lo discuten Solis \& Díaz de León [10]. El primero en utilizar el término panza blanca fue Headden [11], para referirse a los granos que presentan un aspecto moteado a causa de manchas que varían de blanco a blanco amarillento con respecto a las capas externas de la semilla. Manchas de aspecto almidonoso que en algunas ocasiones se presentan en pequeñas áreas, pero pueden llegar a cubrir la mitad o toda la semilla, como lo observaron Sharma et al. [12].

De acuerdo con Aadmodt \& Torrie [13] [14], la panza blanca es una enfermedad fisiológica que disminuye el contenido de proteínas en los granos de trigo, enfermedad que se manifiesta por una condición almidonosa que 
presentan algunos granos, parcial o totalmente en su endosperma. Científicos como Bacci et al. [15] definen a la panza blanca como una alteración de granos, en trigo duro, que afecta la calidad de la harina y, consecuentemente, la calidad de productos como la pasta; mientras que otros como Ammiraju et al. [16] la consideran como un serio desorden en semillas del trigo panadero, trigo duro y triticale, que surge por deficiencia de nitrógeno en el suelo, afectando seriamente el contenido de proteínas del grano y, por tanto, la calidad de la harina en trigo panadero y de la pasta en trigo duro.

Solis \& Díaz de León [10] descubrieron que en trigo duro (Triricum turgidum L. var. Durum), la expresión de la panza blanca se ve favorecida por baja fertilización nitrogenada, uso de variedades sensibles, siembra en la primera quincena de diciembre y por el número de riegos aplicados; no obstante, la panza blanca tiene mayor asociación con la dosis de nitrógeno que con cualquiera de los otros factores mencionados, sin que su incidencia esté correlacionada con el rendimiento. Sin embargo, como lo discuten Solis \& Díaz de León [17], la panza blanca es un desorden fisiológico que afecta al grano de trigo cristalino, con repercusiones negativas en su valor comercial. Este desorden ocurre cuando el cultivo se encuentra en la etapa de llenado de grano, y se identifica por cambios en la coloración del grano y en su composición proteínica.

Partida et al. [18] refieren que las mejores dosis de nitrógeno para disminuir panza blanca del grano de trigo harinero son 110, 140 y $150 \mathrm{~kg}_{\text {de }} \mathrm{N} \mathrm{ha}^{-1}$, ya que éllos descubrieron que la enfermedad disminuyó 41.7, 47.9 y $39.6 \%$, respectivamente, y el rendimiento se incrementó en los respectivos $94.1 \%, 123.4 \%$ y $99.8 \%$, con relación al testigo. Aunque otras dosis de nitrógeno también disminuyen significativamente la panza blanca, pero no son de las mejores para inducir un incremento del rendimiento de grano.

Esta investigación se hizo con la finalidad de conocer el efecto que ocasiona el paclobutrazol en el verdor, altura de plantas y porcentaje de granos con panza blanca de las variedades "Cachanilla F-2000", "Yécora F-70" y "Yécora Rojo”, las cuales se siembran en la región del Valle de Mexicali, Baja California, México.

\section{Materiales y Métodos}

El presente trabajo se realizó en el ejido Nuevo León, del Valle de Mexicali, B. C., con coordenadas 32 24'34" N y 115 11'16" O, donde se utilizaron las variedades de trigo del grupo 1: "Cachanilla F-2000", "Yécora F-70" y "Yécora Rojo". La siembra se llevó a cabo el 17 de Diciembre de 2008 en condiciones de campo abierto y tierra venida, en un diseño de bloques completos al azar con cuatro repeticiones, donde las parcelas experimentales constaron de cuatro surcos de $20 \mathrm{~m} 2$, con separación de $1.0 \mathrm{~m}$ entre camas. El suelo es de textura media con $\mathrm{pH}=7.2, \mathrm{CE}=7.6 \mathrm{dS} \cdot \mathrm{m}^{-1}, \mathrm{~N}$ residual $=147 \mathrm{~kg} \cdot \mathrm{ha}^{-1} \mathrm{y} \mathrm{P}=28.7 \mathrm{~kg} \cdot \mathrm{ha}^{-1}$, se dio el riego por gravedad en presiembra, así como los riegos de auxilio por gravedad necesarios para inducir el mejor crecimiento y desarrollo de las plantas.

Todas las parcelas experimentales fueron manejadas con la fórmula 364-56-00 (147 kg de N residual + 185 a partir de urea y UAN-32; de P fueron 28.7 kg residuals + 27.3 obtenidos de ácido fosfórico). Del total de N aplicado, 35\% se adicionó en el riego de aniego, 32.5\% en el segundo riego de auxilio y 32.5\% en el quinto, o fases $Z_{3}$ y $Z_{7}$ según la escala de Zadoks et al. [19]; en tanto que el total de $P$ se aplicó en el primer riego de auxilio, o fase $\mathrm{Z}_{2}$ de acuerdo con los mismos autores. Las plantas fueron protegidas con la mezcla de herbicidas de $0.6 \mathrm{~L}$ de pinoxaden +10 g de triasulfurón +0.75 L de 2-4-D por hectárea.

Las variables de estudio fueron: verdor, altura de plantas y panza blanca del grano. En tanto que los tratamientos aplicados fueron 0 (testigo), 50, 100, 150, 200, 250, 300 y $350 \mathrm{mg}$ de paclobutrazol (PBZ) $\mathrm{L}^{-1}$ de agua. Las dosis se aplicaron con una bomba manual, en una sola ocasión, al inicio de la etapa de encañe, específicamente cuando las plantas formaron el primer nudo perceptible del tallo principal.

La densidad de población fue de aproximadamente 100 plantas por $\mathrm{m}^{2}$, ya que se utilizaron $46 \mathrm{~kg}$ de semilla por ha, y la siembra se hizo en surcos a doble hilera. El manejo de las plantas se realizó en forma comercial, incluida la fertilización; asimismo, los riegos para incorporar el fertilizante y facilitar la absorción del mismo por parte de las plantas.

Todas las variables se evaluaron en una muestra de 10 plantas seleccionadas al azar. El verdor se midió con un Spad-502, en la parte media de la lámina foliar, específicamente a un lado de la nervadura central, y los datos se recabaron ocho días después de la aplicación del PBZ; la altura se midió a partir de la superficie del suelo hasta la base del raquis de la espiga, y la determinación de panza blanca se hizo con base al peso de $100 \mathrm{~g}$ de grano, de donde se identificaron y pesaron todos aquellos granos con la enfermedad para determinar el respectivo porcentaje. 


\section{Resultados}

Con los datos de verdor en la variedad 'Cachanilla F-2000', se observó que la intensidad de éste fue con una media general de 51.4 Unidades Spad, con respecto a la cual los datos se desviaron 4.5 unidades, lo que a su vez representó el 8.7\% de desviación en relación a la media general. En la Tabla 1 se indican los promedios de verdor, de tal manera que al analizar los valores se detectó que con $100 \mathrm{mg}$ de $\mathrm{PBZ} \mathrm{L}^{-1}$ de agua la característica se incrementó $7.2 \%$ con respecto a lo observado en el testigo; mientras que con 200, 300, 150 ó 350 mg los incrementos fueron de $3.8 \%, 0.9 \%, 0.8 \%$ y $0.7 \%$, en el mismo orden. En la variedad "Yécora F-70", el promedio general y la desviación estándar fueron de 46.3 y 3.9 Unidades Spad, respectivamente, por lo que el coeficiente de variación fue de 8.4\%; sin embargo, el verdor se incrementó en los respectivos $12.1 \%, 10.6 \%, 10.0 \%$, 8.6\%, 7.3\%, 5.5\% y 4.5\% con las dosis de 300, 100, 350, 200, 250, 50 ó $150 \mathrm{mg} \mathrm{de} \mathrm{PBZ} \mathrm{L}^{-1}$ de agua (Tabla 1).

En la variedad "Yécora Rojo", el promedio general de la intensidad del verdor fue de 45.9 Unidades Spad, con una desviación estándar de 4.3 unidades y un coeficiente de variación de $9.4 \%$; no obstante, dicha característica también se incrementó en $19.1 \%, 8.0 \%, 5.3 \%, 4.9 \%, 4.6 \%, 3.4 \%$ y 2.0\% (Tabla 1) con las dosis de 50, 250, 150, 200, 350, 300, ó 100 mg de PBZ, en el mismo orden. La respuesta en el verdor indica que el PBZ ocasionó mayor eficiencia fotosintética en las plantas de las tres variedades.

De manera general, la variedad "Cachanilla F-2000" fue 44.6\% y 25.3\% más alta que las variedades "Yécora F-70” y "Yécora Rojo” (Tabla 2); sin embargo, al analizar por separado el promedio de cada cultivar se notaron diferencias de 6.0\%, 6.3\% y 6.3\% entre la altura del testigo de la variedad "Cachanilla F-2000" y la de las plantas cultivadas con 50, 100 y $150 \mathrm{mg}$ de $\mathrm{PBZ} \mathrm{L}^{-1}$ de agua, pero las diferencias fueron de mayor magnitud (9.4, $10.7 \%, 10.9 \%$ y 13.0\%) entre las que recibieron 200, 250, 300 ó $350 \mathrm{mg}$ de PBZ y las plantas testigos. En el cultivar "Yécora F-70" la mayor disminución (10 \%) ocurrió con la dosis de $150 \mathrm{mg}$ de PBZ, en tanto que con 50, 100, 200, 250, 300 y $350 \mathrm{mg}$ de PBZ las respectivas disminuciones fueron de $0.4 \%, 1.8 \%, 4.8 \%, 2.3 \%$, 2.9\% y 1.5\%. Mientras que en la variedad "Yécora Rojo" las disminuciones de altura oscilaron de $7.9 \%$ - 17.0\% con las dosis de 50 a $350 \mathrm{mg}$.

La panza blanca disminuyó hasta cero en las variedades "Yécora F-70” y "Yécora Rojo” con todas las dosis de PBZ (Tabla 3); sin embargo, en el cultivar "Cachanilla F-2000” las disminuciones oscilaron de 48.5\% - 73.4\% con todas las dosis utilizadas en relación a los porcentajes observados en los granos cosechados de las plantas testigo.

La correlación de las dosis del PBZ con el verdor sólo fue positiva en la variedad "Yécora F-70" con $\mathrm{r}=0.72$ ( $\mathrm{p}$ $=0.0002)$; con la altura de las plantas fue $r=-0.94(p=0.0005)$ en el cultivar "Cachanilla F-2000" y de $r=$ -0.73 ( $p=0.0002)$ en la variedad "Yécora Rojo", mientras que en "Yécora F-70" también fue negativa pero estadísticamente no significativa; la relación entre dichas dosis y los granos con panza blanca fue $\mathrm{r}=-0.65,-0.64$ y -0.64 ( $p=0.0815,0.0825$ y 0.0822) en los cultivares “Cachanilla F-2000", "Yécora F-70” y "Yécora Rojo",

Tabla 1. Verdor de las plantas de trigo de tres variedades tratadas con PBZ al inicio de la etapa de encañe (formación de tallos).

\begin{tabular}{cccc}
\hline $\begin{array}{c}\text { Tratamientos } \\
\left(\mathrm{mg} \cdot \mathrm{L}^{-1} \text { de agua) }\right.\end{array}$ & $\begin{array}{c}\text { Cachanilla F-2000 } \\
\text { (Unidades Spad) }\end{array}$ & $\begin{array}{c}\text { Yécora F-70 } \\
\text { (Unidades Spad) }\end{array}$ & $\begin{array}{c}\text { Yécora Rojo } \\
\text { (Unidades Spad) }\end{array}$ \\
\hline Testigo (0) & $50.62 \mathrm{~b}$ & $43.17 \mathrm{~b}$ & $43.34 \mathrm{~b}$ \\
50 & $50.59 \mathrm{~b}$ & $45.56 \mathrm{ab}$ & $51.64 \mathrm{a}$ \\
100 & $54.29 \mathrm{a}$ & $47.75 \mathrm{ab}$ & $44.19 \mathrm{~b}$ \\
150 & $51.02 \mathrm{ab}$ & $45.11 \mathrm{ab}$ & $45.63 \mathrm{ab}$ \\
200 & $52.55 \mathrm{ab}$ & $46.87 \mathrm{ab}$ & $45.45 \mathrm{~b}$ \\
250 & $50.32 \mathrm{~b}$ & $46.34 \mathrm{ab}$ & $46.82 \mathrm{ab}$ \\
300 & $51.08 \mathrm{ab}$ & $48.38 \mathrm{a}$ & $44.83 \mathrm{~b}$ \\
350 & $50.96 \mathrm{~b}$ & $47.47 \mathrm{ab}$ & $45.33 \mathrm{~b}$ \\
DMSH & 3.63 & 5.21 & 6.129 \\
\hline
\end{tabular}

Medias con letras diferentes en la columna son estadísticamente diferentes (Tukey, $\leq 0.05$ ). 
Tabla 2. Altura de plantas de trigo de tres variedades tratadas con PBZ al inicio de la etapa de encañe (formación de tallos).

\begin{tabular}{cccc}
\hline $\begin{array}{c}\text { Tratamientos } \\
\left(\mathrm{mg} \cdot \mathrm{L}^{-1} \text { de agua }\right)\end{array}$ & $\begin{array}{c}\text { Cachanilla } 2000 \\
(\mathrm{~cm})\end{array}$ & $\begin{array}{c}\text { Yécora F-70 } \\
(\mathrm{cm})\end{array}$ & $\begin{array}{c}\text { Yécora Rojo } \\
(\mathrm{cm})\end{array}$ \\
\hline Testigo (0) & $86.75 \mathrm{a}$ & $60.00 \mathrm{a}$ & $69.25 \mathrm{a}$ \\
50 & $81.50 \mathrm{ab}$ & $59.75 \mathrm{ab}$ & $63.75 \mathrm{ab}$ \\
100 & $81.25 \mathrm{ab}$ & $58.90 \mathrm{ab}$ & $61.25 \mathrm{~b}$ \\
150 & $81.25 \mathrm{ab}$ & $54.00 \mathrm{~b}$ & $59.00 \mathrm{~b}$ \\
200 & $78.58 \mathrm{ab}$ & $57.12 \mathrm{ab}$ & $61.00 \mathrm{~b}$ \\
250 & $77.50 \mathrm{ab}$ & $58.60 \mathrm{ab}$ & $57.50 \mathrm{~b}$ \\
300 & $77.25 \mathrm{ab}$ & $58.25 \mathrm{ab}$ & $59.25 \mathrm{~b}$ \\
350 & $75.50 \mathrm{~b}$ & $59.10 \mathrm{ab}$ & $60.75 \mathrm{~b}$ \\
DMSH & 11.19 & 5.67 & 7.99 \\
\hline
\end{tabular}

Medias con letras diferentes en la columna son estadísticamente diferentes (Tukey, $\alpha \leq 0.05$ ).

Tabla 3. Gramos de granos con panza blanca en tres variedades de trigo tratadas con paclobutrazol al formar el primer nudo del tallo.

\begin{tabular}{cccc}
\hline \multirow{2}{*}{$\begin{array}{c}\text { Dosis de PBZ } \\
\left(\mathrm{mg} \cdot \mathrm{L}^{-1} \text { de agua }\right)\end{array}$} & \multicolumn{2}{c}{ Gramos de granos con panza blanca por variedad } \\
\cline { 2 - 4 } Testigo (0) & Cachanilla F-2000 & Yécora F-70 & Yécora Rojo \\
\hline 50 & $8.45 \mathrm{a}$ & $0.77 \mathrm{a}$ & $0.80 \mathrm{a}$ \\
100 & $4.35 \mathrm{~b}$ & $0.00 \mathrm{~b}$ & $0.12 \mathrm{~b}$ \\
150 & $3.22 \mathrm{~b}$ & $0.17 \mathrm{~b}$ & $0.00 \mathrm{~b}$ \\
200 & $2.32 \mathrm{~b}$ & $0.00 \mathrm{~b}$ & $0.00 \mathrm{~b}$ \\
250 & $2.25 \mathrm{~b}$ & $0.00 \mathrm{~b}$ & $0.00 \mathrm{~b}$ \\
300 & $2.80 \mathrm{~b}$ & $0.00 \mathrm{~b}$ & $0.00 \mathrm{~b}$ \\
350 & $2.52 \mathrm{~b}$ & $0.00 \mathrm{~b}$ & $0.00 \mathrm{~b}$ \\
DMSH & $3.42 \mathrm{~b}$ & $0.00 \mathrm{~b}$ & $0.00 \mathrm{~b}$ \\
& 3.95 & 0.13 & 0.11 \\
\hline
\end{tabular}

Medias con diferentes en la columna son estadísticamente diferentes (Tukey, $\alpha \leq 0.05$ ).

respectivamente, en tanto que la correlación de la altura con los granos con panza blanca fue $\mathrm{r}=0.73(\mathrm{p}=$ 0.0002) en "Cachanilla F-2000", $r=0.90(p=0.0001)$ en "Yécora Rojo" y $r=0.41(p=0.0923)$ en "Yécora F-70"; la asociación entre la clorofila y los granos con panza blanca fue negativa en los tres cultivares, pero sólo fue significativa ( $\mathrm{p}=0.0003$ ) en "Yécora F-70" con $\mathrm{r}=-0.69$. En todos los casos, la relación del verdor con la altura de plantas no fue significativa estadísticamente.

\section{Discusión}

Los resultados en el verdor de las tres variedades tienen relación con lo descubierto por Ali [9] en plantas de pepino, ya que también observó incremento de la concentración de clorofila y mayor eficiencia fotosintética en plantas obtenidas a partir de semillas remojadas en solución con paclobutrazol.

Los resultados obtenidos en altura de los tres cultivares (“Cachanilla F-2000”, "Yécora F-70” y "Yécora Rojo") mostraron relación con los obtenidos por Espindula et al. [20], puesto que éllos observaron que la altura del trigo disminuye ligeramente al aplicar PBZ en dosis de 40, 80 ó $120 \mathrm{~g} \cdot \mathrm{ha}^{-1}$. El incremento en 1.7\% de altura de 


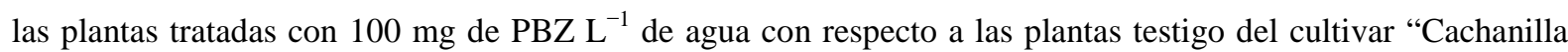
F-2000”, coincide con los resultados de Velázquez et al. [8], ya que éstos observaron incrementos en la altura de plantas de tomate y chiles, respectivamente, cuando aplicaron dosis de 250, 300 ó $350 \mathrm{mg}$ de $\mathrm{PBZ} \mathrm{L}^{-1}$ de agua. Asimismo, los resultados en las variedades "Yécora F-70" y "Yécora Rojo" se relacionan con los de los mismos autores, ya que éstos también encontraron las respectivas disminuciones de altura en tomate y diferentes tipos de chiles, cuando aplicaron dosis de 100, 150 ó 200 mg de PBZ.

Las disminuciones de panza blanca quizá se debieron a que las plantas tratadas con PBZ produjeron mayor número de raíces y más largas que las testigo, como lo descubrieron Watson [3] y Litembani \& Taylor [4] al observar que el PBZ incrementa el crecimiento de raíces o aumenta la relación de raíces por brotes en plantas de durazno (Prunus persica L.), o como lo descubrió Ruter [5] en Pyracantha y Juniperus que produjeron más raíces al ser tratadas con PBZ.

Dicho incremento de raíces también lo descubrieron Partida et al. [7] en plantas de pimiento morrón (Capsicum annuum) variedad 'California Wonder' y en berenjena (Solanum melongena) varidad "Dalia”, ya que en la primera especie, la dosis de $150 \mathrm{mg}$ de $\mathrm{PBZ} \mathrm{L}^{-1}$ de agua ocasionó incremento de raíces hasta 1.1 veces más en longitud, 3.7 veces en materia fresca y 13 veces en materia seca; mientras que en berenjena produjo incremento en 1.3 veces la materia fresca y en $71 \%$ la materia seca de raíces.

De tal manera que con raíces más abundantes y largas las plantas exploraron más suelo y absorbieron más nitrógeno, magnesio, calcio, cobre, zinc, entre otros nutrientes, para metabolizar más clorofila, como lo notó Ali [9] en plantas de pepino tratadas con PBZ, asimismo, más proteínas que almidón para disminuir la panza blanca en el grano, ya que según Aadmodt \& Torrie, [13] [14], esta enfermedad es fisiológica y se debe a menos proteínas en los granos de trigo, lo que de acuerdo con Solis \& Díaz de León [17], ocurre cuando el cultivo se encuentra en la etapa de llenado del grano de trigo Triticum turgidum L. var. durum.

Además, los resultados de Solis \& Díaz de León [10], indican que en trigo duro (Triricum turgidum L. var. Durum) la panza blanca se favorece por baja concentración de nitrógeno en el suelo; lo que se corroboró por Ammiraju et al. [16], al descubrir que la panza blanca surge por deficiencia de nitrógeno en el suelo, afectando el contenido de proteínas del grano y, por tanto, la calidad de la harina en trigo panadero y de la pasta en trigo duro.

\section{Conclusion}

Los resultados obtenidos indican que el PBZ puede ser utilizado para incrementar el proceso fotosintético, ya que el verdor tendió a incrementarse en las hojas de los tres cultivares (“Cachanilla F-2000”, "Yécora F-70” y "Yécora Rojo"), y aunque con la mayoría de las dosis aplicadas la altura tendió a disminuir ligeramente en dichas variedades, los efectos también se manifestaron en la disminución de la panza blanca del grano, lo que permite sugerir que el PBZ y su aplicación al inicio del encañe son dos tecnologías que se pueden utilizar para mejorar la calidad del grano de trigos harineros y duros.

\section{References}

[1] Ochoa, B.R. and Ortega, R.C. (2009) Reservas alimentarias físicas y virtuales. Claridades Agropecuarias, 189, 3-14.

[2] Clouse, S.D. and Sasse, J.M. (1998) Brassinosteroids: Essential Regulators of Plant Growth and Development. Annual Review of Plant Physiology and Plant Molecular Biology, 49, 427-451. http://dx.doi.org/10.1146/annurev.arplant.49.1.427

[3] Watson, G.W. (1996) Tree root System Enhancement with Paclobutrazol. Journal Arboriculture, 22, 211-217.

[4] Litembani, S. and Taylor, B.H. (1989) Growth and Development of Young Peach Trees as Influenced by Foliar Sprays of Paclobutrazol or XE-1019. HortScience, 24, 65-68.

[5] Ruter, J.M. (1994) Growth and Landscape Establishment of Pyracantha and Juniperus after Application of Paclobutrazol. HortScience, 29, 1318-1320.

[6] Osuna, G.J.A., Baez, S.R., Medina, U.V.M. and Chávez, C.X. (2001) Residualidad de paclobutrazol en frutos de mango (Mangifera indica L.) cultivar Tommy Taquín. Revista Chapingo Serie Horticultura, 7, 275-282.

[7] Partida, R.L., Velázquez, A.T.J., Acosta, V.B., Ayala, T.F., Díaz, V.T., Inzunza, C.J.F. and Cruz, O.J.E. (2007) Paclobutrazol y crecimiento de raíz y parte aérea de plántulas de pimiento morrón y berenjena. Revista Fitotecnia Mexicana, 30, 145-149.

[8] Velázquez, A.T.J., Partida, R.L., Acosta, V.B. and Ayala, T.F. (2008) Producción de plantas de tomate y chile apli- 
cando paclobutrazol al follaje. Universidad y Ciencia, 24, 21-28.

[9] Ali, A.R. (2009) Improving Germination Performance and Chilling Tolerance in Cucumber Seedlings with Paclobutrazol. Internacional Journal of Vegetable Science, 15, 173-184. http://dx.doi.org/10.1080/19315260802685768

[10] Solis, M.E. and Díaz de León, T.J.G. (2001) Efecto de los factores controlables de la producción sobre el rendimiento y la panza blanca del grano en trigo duro. Terra Latinoamericana, 19, 375-383.

[11] Headden, W.P. (1916) Yellow-Berry in Wheat: Its Cause and Prevention. Published by the Agricultural Experiment Station Fort Collins, Colorado. Bulletin 205, 1-38. http://babel.hathitrust.org/cgi/pt?id=uiug.30112019444246;view=1up;seq=7

[12] Sharma, G.C., Paul, A.D. and Bietz, J.A. (1983) Nitrogen Fertilization Effects and Anatomical, Protein and Amino Acid Characteristics of Yellow Berry in Triticale. Crop Science, 23, 699-703.

[13] Aamodt, O.S. and Torrie, J.H. (1935) Kernel Texture as an Indicator of Quality in Hard Red Spring Wheats. Canadian Journal of Research Section C Botanical Sciences, 13, 79-88. http://dx.doi.org/10.1139/cjr35c-005

[14] Aamodt, O.S. and Torrie, J.H. (1935) Studies on the Inheritance of the Relation between Kernel Texture and Protein Content in Several Spring Wheat Crosses. Canadian Journal of Research Section C Botanical Sciences, 13, $202-219$. http://dx.doi.org/10.1139/cjr35c-016

[15] Bacci, L., Rapi, B. and Colucci, F. (2002) An Evaluation Technique of Yellow-Berry in Durum Wheat Seeds. Proceedings of the World Congress of Computers in Agriculture and Natural Resources, Iguacu Falls, 13-15 March 2002, 62-69. http://dx.doi.org/10.13031/2013.8313

[16] Ammiraju, J.S.S., Dholakia, B.B., Jawdekar, G., Santra, D.K., Gupta, V.S., Roder, M.S., Singh, H., Lagu, M.D., Dhaliwal, H.S., Rao, V.S. and Ranjekar, P.K. (2002) Inheritance and Identification of DNA Markers Associated with Yellow Berry Tolerante in Wheat (Triticum aestivum L.). Euphytica, 123, 229-233. http://dx.doi.org/10.1023/A:1014914101037

[17] Solis, M.E., Espinoza, T.E., Ramírez, R.A., Ríos, R.S.A. and Grageda, C.O.A. (2009) El carácter panza blanca del grano en trigo cristalino. Factores que influyen en su control, Folleto Científico No. 2, 8 p.

[18] Partida, R.L., Velázquez, A.T.J., Díaz, V.T., Acosta, V.B. and Inzunza, C.J.F. (2007) Nitrógeno, disminución de panza blanca del grano y aumento del rendimiento del trigo (Triticum aestivum L.). Revista Electrónica Granma Ciencia, 11, 1-9.

[19] Zadock, J.C., Chang, T.T. and Konzak, C.F. (1974) A Decimal Code for the Growth Stages of Cereals. Weed Research, 14, 415-421. http://dx.doi.org/10.1111/j.1365-3180.1974.tb01084.x

[20] Espindula, M.C., Rocha, V.S., Grossi, J.A.S., Souza, M.A., Souza, L.T. and Favarato, L.F. (2009) Use of Growth Retardants in Wheat. Planta Daninha, 27, 379-387. http://dx.doi.org/10.1590/S0100-83582009000200022 